LA-UR-98-3410

CPHT-S638.0898

\title{
Fractal Geometry of Quantum Spacetime at Large Scales
}

\author{
Ignatios Antoniadis \\ Centre de Physique Théorique, Ecole Polytechnique, \\ F-91128 Palaiseau, France \\ antoniad@cpht.polytechnique.fr \\ Pawel O. Mazur \\ Dept. of Physics and Astronomy, University of S. Carolina, \\ Columbia, SC 29208 USA \\ mazur@psc.psc.sc.edu \\ and \\ Emil Mottola \\ Theoretical Division, Los Alamos National Laboratory, \\ MS B285, Los Alamos, New Mexico 87545 USA \\ emil@pion.lanl.gov
}

\begin{abstract}
We compute the intrinsic Hausdorff dimension of spacetime at the infrared fixed point of the quantum conformal factor in $4 \mathrm{D}$ gravity. The fractal dimension is defined by the appropriate covariant diffusion equation in four dimensions and is determined by the coefficient of the Gauss-Bonnet term in the trace anomaly to be generally greater than 4 . In addition to being testable in simplicial simulations, this scaling behavior suggests a physical mechanism for the screening of the effective cosmological 'constant' and inverse Newtonian coupling at very large distance scales, which has implications for the dark matter content and large scale structure of the universe.
\end{abstract}


The concept of gauge invariance, and indeed the term itself first entered physics through the attempts of Weyl to extend the general coordinate invariance of relativity to scale or conformal symmetry. Although these early explorations led to models that were quickly ruled out by observations, scale invariance has played a central role in several later developments, most notably the impressive success of renormalization group techniques in determining the critical behavior of second order phase transitions in a wide variety of statistical systems. More recently, conformal symmetry was rejoined with gravity in the quantum Liouville theory of 2D surfaces where it has made possible the analytic solution of the model [1].

The key observation which made possible this progress in 2D gravity is that the 'classical' action is not complete, but rather must be augmented by an additional term to account for the quantum trace anomaly of matter in a general curved background. Since this additional term is infrared relevant (strictly speaking, marginal) in the language of the renormalization group, it cannot be neglected at large distances, where indeed it changes completely the predictions of the classical theory and produces nontrivial scaling exponents which are determined by the trace anomaly central charge [1]. These analytic predictions of scaling exponents have been confirmed by numerical simulations on dynamically triangulated lattices [2].

In four spacetime dimensions the quantum trace anomaly of massless conformal fields requires the introduction of an additional term in the effective action by precisely analogous reasoning [3, [4]. This term is nonlocal when written in a generally covariant form, becoming local only in the conformal gauge parameterization. Let us be very clear that because of the existence of nontrace modes in the four dimensional metric tensor, this anomaly generated term is only one of many in the full effective action and therefore gives only strictly limited information about the quantum effects of matter in curved spacetime. Certainly it cannot be used to extract any information about Ward identities satisfied by products of untraced energy-momentum tensors, or any other quantity which depends on the nontrace (i.e. ordinary graviton) dynamics of the metric. Nor can this effective action be trusted to describe the physics of the ultrashort Planck scale, where the very notion of the spacetime metric itself most likely becomes useless. Nevertheless, this term in the effective action reproduces the full dependence on the trace of the metric required by the trace anomaly of massless degrees of freedom, and just as in the 2D Liouville theory it is strictly infrared marginal under conformal transformations. Hence, it is sharply distinguished from any of the myriad other terms in the quantum effective action of gravity by the fact that it does not decouple at low energies and hence its effects cannot be neglected on large distance scales.

The new term in the effective action of $4 \mathrm{D}$ gravity produces an important qualitative departure from the classical Einstein theory, namely, the addition of a new degree of freedom in the trace or conformal sector of the metric. As is well known, the Einstein theory describes only spin- 2 propagating degrees of freedom, its spin- 0 or trace component being completely frozen by the classical constraints [5]. Since these classical constraints of general relativity are necessarily modified by the trace anomaly, the new fourth order term in the quantum effective action allows the scalar spin- 0 component of the metric tensor to become dynamical and fluctuate as well. However, even in the quantum theory the constraints of diffeomorphism invariance are quite stringent and serve to eliminate all the purely local propagating degrees of freedom in the scalar sector (negative metric or otherwise), leaving behind only a single new global degree of freedom [6]. This is in marked contrast to local fourth order derivative modifications of the Einstein action which describe local propagating modes that lead to 
violations of energy positivity and unitarity.

Because the fluctuations of the conformal factor are global in character the classical Einstein theory should remain largely intact at all scales intermediate between the extreme ultraviolet Planck scale and the extreme infrared horizon scalel. In particular, there is no new local gravitationally coupled spin-0 degree of freedom predicted by the new term, and therefore no conflict with observational bounds on such dilaton-like scalar particles. However we have argued elsewhere that the fluctuations of the conformal factor become relevant at the horizon scale of the classical expanding universe $[4,8]$. In fact, the effective theory of the trace anomaly induced action predicts a conformally invariant fixed point of gravity at very large distance scales where the new term dominates the classical Einstein term, scale invariance is restored, and the anomalous dimensions of the Einstein and cosmological terms can be computed in closed form in terms of a single anomaly coefficient $Q^{2}$, just as in the 2D Liouville theory. In a sense this restoration of global conformal symmetry at the infrared fixed point is a realization of Weyl's original idea of 'gauge' invariance in gravity, where the rigid distance standard of Newtonian physics, which is preserved in Einstein's theory, gives way to complete invariance under distance reparameterizations, in the same way as in the theory of second order phase transitions at the critical temperature.

In previous letters we have discussed the predictions of the anomalous dimensions and scaling behavior of the infrared fixed point of the conformal factor, both for numerical simulations on dynamically triangulated lattices, and on the spectrum and statistics of the cosmic microwave background radiation [0,8]. In this Letter we wish to expose two additional effects, namely the fractal dimension of spacetime and the effective running of the Newtonian and cosmological 'constants' at large distances, both of which are implied by the anomalous dimensions and scaling exponents computed previously. These provide additional predictions for the simplicial simulations and suggest additional implications for the dark matter content and very large scale structure of the universe, which we discuss below.

\section{Hausdorff Dimension.}

In order to define a Hausdorff dimension for spacetime one needs to relate the geodesic distance $\ell\left(x, x^{\prime}\right)$ between points $x$ and $x^{\prime}$ to the volume $V_{\ell}$ enclosed by the spherical surface with radius equal to $\ell$. The scaling relation between the two,

$$
V_{\ell} \sim \ell^{d_{H}}
$$

for large $\ell$ defines the intrinsic Hausdorff dimension $d_{H}$ of the space.

In $2 \mathrm{D}$ quantum gravity the definition of distance that seems to be most appropriate is that defined by the heat kernel of the Laplacian operator $\square$, i.e. [9]

$$
K_{2}\left(x, x^{\prime} ; s ; g\right)=<x|\exp (-s \square)| x^{\prime}>\text {. }
$$

This quantity has the advantage of being manifestly covariant, and since $\square$ in the metric $g$ is an operator with well-defined scaling dimensions under conformal transformations,

\footnotetext{
${ }^{1}$ An exception to this may be in very strong gravitational fields, such as in black holes.
} 


$$
\square=e^{-2 \sigma} \bar{\square} \quad \text { for } \quad g_{a b}=e^{2 \sigma} \bar{g}_{a b},
$$

the heat kernel $K_{2}$ has a short distance expansion whose anomalous scaling behavior may be calculated easily by standard techniques. We define the average geodesic length squared that a scalar particle will have diffused after proper time $s$ by

$$
\ell_{s}^{2} \equiv \frac{1}{V}\left\langle\int d^{2} x \sqrt{g} \int d^{2} x^{\prime} \sqrt{g}^{\prime} \ell^{2}\left(x, x^{\prime} ; g\right) K_{2}\left(x, x^{\prime} ; s ; g\right)\right\rangle_{V}
$$

where the average is with respect to the fixed volume Liouville partition function. By expanding the heat kernel $K_{2}$ in a power series in $s$ it is easy to see that

$$
\ell_{s}^{2} \sim s \quad \text { as } \quad s \rightarrow 0
$$

which is the standard result for a particle undergoing Brownian motion.

On the other hand the probability that the particle will come back to a small region within $\epsilon$ of its starting point $x_{0}$ after proper time $s$ is

$$
\left\langle\int d^{2} x \sqrt{g} K_{2}\left(x, x_{0} ; s ; g\right) f_{\epsilon}\left(x, x_{0}\right)\right\rangle_{V}=\left\langle\int d^{2} x \sqrt{g}\left(1-s \square+\mathcal{O}\left(s^{2}\right)\right) f_{\epsilon}\left(x, x_{0}\right)\right\rangle_{V}
$$

where $f_{\epsilon}\left(x, x^{\prime}\right)$ is any smooth function with support only for $\ell\left(x, x^{\prime}\right) \sim\left|x-x^{\prime}\right| \lesssim \epsilon$ normalized by $\int d^{2} x \sqrt{g} f_{\epsilon}\left(x, x^{\prime}\right)=1$, i.e. it approaches the delta function, $\frac{1}{\sqrt{g}} \delta^{2}\left(x-x^{\prime}\right)$ as $\epsilon \rightarrow 0$. From this factor of $\frac{1}{\sqrt{g}}$ and (3) it follows that the operator multiplying the term linear in $s$ in (6) is a density of weight -1 in the Liouville theory, 2 and therefore has a well-defined scaling behavior with volume. Hence,

$$
s\left\langle\int d^{2} x \sqrt{g} \square f_{\epsilon}\left(x, x_{0}\right)\right\rangle_{V}=s\left\langle\int d^{2} x \sqrt{\bar{g}} e^{2 \alpha_{-1} \sigma} \bar{\square} \bar{f}_{\epsilon}\left(x, x_{0}\right)\right\rangle_{V} \sim s V^{\frac{\alpha_{-1}}{\alpha_{1}}},
$$

where the finite volume scaling of the last proportionality follows by a simple constant shift in the Liouville field, $\sigma \rightarrow \sigma+\sigma_{0}$, and the $\alpha_{n}$ are scaling dimensions, given in the Liouville theory by the general formula,

$$
\alpha_{n}=n+\frac{\alpha_{n}^{2}}{Q^{2}}=\frac{2 n}{1+\sqrt{1-\frac{4 n}{Q^{2}}}}
$$

in terms of $Q^{2}$ which is determined in terms of the matter central charge (anomaly coefficient) $c_{m}$ by

$$
Q^{2}=\frac{25-c_{m}}{6}, \quad D=2
$$

Since the return probability (6) is independent of a rescaling of the total volume $V \rightarrow \lambda V$ for small $s$ (before the particle can feel the effects of the finite volume which is assumed to

\footnotetext{
${ }^{2}$ Here we follow the standard conventions in $D=2$ in which the conformal weights are chiral and hence should be doubled to compare with the corresponding weights in $D=4$.
} 
be very large, i.e. lattice finite volume effects are assumed to be irrelevant), it follows that $s$ must scale like

$$
s \rightarrow \lambda^{-\frac{\alpha_{-1}}{\alpha_{1}}} s
$$

under a global volume scaling. From this result and (5) we conclude that

$$
\ell_{s}^{2} \sim s \sim V_{\ell}^{-\frac{\alpha_{-1}}{\alpha_{1}}}
$$

Inverting this relation for $V_{\ell}$ in terms of $\ell$ and using (1) gives finally the Hausdorff dimension,

$$
d_{H}=-2 \frac{\alpha_{1}}{\alpha_{-1}}=2 \frac{\sqrt{25-c_{m}}+\sqrt{49-c_{m}}}{\sqrt{25-c_{m}}+\sqrt{1-c_{m}}} \geq 2, \quad D=2
$$

for 2D gravity [9]. This formula predicts $d_{H}=4$ for pure $2 \mathrm{D}$ gravity (i.e. $\left.c_{m}=0\right), d_{H}=$ $2(1+\sqrt{2})$ at the limit of its validity for $c_{m}=1$ and $d_{H} \rightarrow 2$, its classical value, in the classical limit, $c_{m} \rightarrow-\infty$, or $Q^{2} \rightarrow+\infty$, indicating that the geometries become smooth and classical in that limit. A summary of the agreement between (12) and numerical simulations for various values of $c_{m}$ is given in refs. 10.

A precisely parallel computation will now be given for four dimensional gravity at the infrared fixed point determined by the fluctuations of the quantum conformal factor. First we note that we cannot use the second order operator $\square$ in four dimensions since it no longer has a well-defined conformal weight. Adding a $-R / 6$ correction does not help since it operates on conformal scalars which have weight one in $D=4$ (not zero as in $D=2$ ). Thus the calculation above will not go through as it did in $D=2$ for any second order scalar operator in $D=4$. Instead we suggest that the appropriate differential operator with which to define an invariant diffusion length is

$$
\Delta_{4} \equiv \square^{2}+2 R^{a b} \nabla_{a} \nabla_{b}-\frac{2}{3} R \square+\frac{1}{3}\left(\nabla^{a} R\right) \nabla_{a}
$$

which satisfies

$$
\Delta_{4}=e^{-4 \sigma} \bar{\Delta}_{4}
$$

for $g_{a b}=e^{2 \sigma} \bar{g}_{a b}$. Hence like $\square$ in $D=2, \Delta_{4}$ in $D=4$ transforms homogeneously under local conformal rescalings of the metric and operates on scalar functions with zero conformal weight. It is also precisely the operator which enters the anomaly induced action in four dimensions, just as $\square$ does in two dimensions. Hence we propose to define the heat kernel,

$$
K_{4}\left(x, x^{\prime} ; s ; g\right) \equiv<x\left|\exp \left(-s \Delta_{4}\right)\right| x^{\prime}>
$$

and the average geodesic length after proper time $s$ by

$$
\ell_{s}^{4} \equiv \frac{1}{V}\left\langle\int d^{4} x \sqrt{g} \int d^{4} x^{\prime} \sqrt{g}^{\prime} \ell^{4}\left(x, x^{\prime} ; g\right) K_{4}\left(x, x^{\prime} ; s ; g\right)\right\rangle_{V}
$$

The angular brackets here denote the quantum expectation value in the finite volume Euclidean partition function $Z(\kappa ; V)$, namely, 


$$
\langle\mathcal{O}\rangle_{V}=\frac{1}{Z(\kappa ; V)} \int[\mathcal{D} \sigma] \mathcal{O} \exp \left(-S_{4}[\sigma]-\frac{1}{2 \kappa} S_{2}[\sigma]\right) \delta\left(S_{0}[\sigma]-V\right)
$$

where

$$
S_{0}[\sigma]=\int d^{4} x \sqrt{g}=\int d^{4} x \sqrt{\bar{g}} e^{4 \sigma} .
$$

is the classical Euclidean four-volume,

$$
S_{2}[\sigma]=-\int d^{4} x \sqrt{g} R=6 \int d^{4} x \sqrt{\bar{g}} e^{2 \sigma}\left[\bar{\square} \sigma+(\bar{\nabla} \sigma)^{2}-\frac{\bar{R}}{6}\right]
$$

is the classical Einstein-Hilbert action, and

$$
S_{4}[\sigma]=\frac{Q^{2}}{(4 \pi)^{2}} \int d^{4} x \sqrt{g}\left[\sigma \Delta_{4} \sigma+\frac{1}{2}\left(G-\frac{2}{3} \square R\right) \sigma\right],
$$

is the new term in the effective action required by the trace anomaly. We recall from refs. [4,7] that the classical expressions for $S_{0}$ and $S_{2}$ are gravitationally dressed so that their classical scaling dimensions (4 and 2 respectively) are modified at the quantum level. In the expression for $S_{4}, Q^{2}$ is the coefficient of the Gauss-Bonnet term $G$ in the four dimensional conformal anomaly, given by

$$
Q^{2}=\frac{1}{180}\left(N_{S}+\frac{11}{2} N_{W F}+62 N_{V}-28\right)+Q_{\text {grav }}^{2},
$$

in terms of the number of free scalar $\left(N_{S}\right)$, Weyl fermion $\left(N_{W F}\right)$ and vector $\left(N_{V}\right)$ fields, while the -28 and $Q_{\text {grav }}^{2}$ are the contributions of the spin-0 conformal factor and spin-2 graviton fields of the metric itself.

Now by expanding the heat kernel $K_{4}$ in (15) and (16) in powers of $s$ we obtain

$$
\ell_{s}^{4} \sim s \quad \text { as } \quad s \rightarrow 0
$$

in place of (5). Defining next the return probability analogous to (6) in $D=4$,

$$
\left\langle\int d^{4} x \sqrt{g} K_{4}\left(x, x_{0} ; s ; g\right) f_{\epsilon}\left(x, x_{0}\right)\right\rangle_{V}=\left\langle\int d^{4} x \sqrt{g}\left(1-s \Delta_{4}+\mathcal{O}\left(s^{2}\right)\right) f_{\epsilon}\left(x, x_{0}\right)\right\rangle_{V}
$$

where $f_{\epsilon}$ approaches $\frac{1}{\sqrt{g}} \delta^{4}\left(x, x^{\prime}\right)$ as $\epsilon \rightarrow 0$, we find that we need the conformal scaling exponents $\beta_{0}$ and $\beta_{8}$, corresponding to the volume operator (a density of weight 4 , codimension $\bar{\Delta}=$ $4-w=0$ ) and $\Delta_{4}$ operator (a density of weight $w=-4$, codimension $\bar{\Delta}=4-(-4)=8$ ) respectively. Indeed for any operator with well-defined scaling codimension $\bar{\Delta}$ in the absence of gravitational fluctuations we have found previously that [7]

$$
\left\langle\mathcal{O}_{\bar{\Delta}}\right\rangle_{V} \sim V^{1-\frac{\Delta}{4}}=V^{\frac{\beta_{\bar{\Delta}}}{\beta_{0}}} .
$$

These scaling exponents $\beta_{\bar{\Delta}}$ are given by the general quadratic relation,

$$
\beta_{\bar{\Delta}}=4-\bar{\Delta}+\frac{\beta_{\bar{\Delta}}^{2}}{2 Q^{2}},
$$


analogous to (8), in the notation of [7], where $\bar{\Delta}=4-w$ is the codimension of the operator with conformal weight $w$ (analogous to $2-2 n$ in $D=2$, so that $2 \alpha_{n}$ is replaced by $\beta_{\bar{\Delta}}$ in $D=4$ ). Hence we find that the term linear in $s$ in (23) scales like

$$
\left\langle\int d^{4} x \sqrt{g} s \Delta_{4} f_{\epsilon}\left(x, x_{0}\right)\right\rangle_{V}=\left\langle\int d^{4} x \sqrt{\bar{g}} s \bar{\Delta}_{4} e^{\beta_{8} \sigma} \bar{f}_{\epsilon}\left(x, x_{0}\right)\right\rangle_{V} \sim s V^{\frac{\beta_{8}}{\beta_{0}}}
$$

so that $s$ must scale like

$$
s \rightarrow \lambda^{-\frac{\beta_{8}}{\beta_{0}}} s
$$

under a global volume scaling, $V \rightarrow \lambda V$ in $D=4$. Combining this result with (22) we obtain

$$
\ell_{s}^{4} \sim s \sim V_{\ell}^{-\frac{\beta_{8}}{\beta_{0}}}
$$

so that solving for $V_{\ell}$ in terms of $\ell$ and using (1) and (25) yields the Hausdorff dimension,

$$
d_{H}=-4 \frac{\beta_{0}}{\beta_{8}}=4 \frac{1+\sqrt{1+\frac{8}{Q^{2}}}}{1+\sqrt{1-\frac{8}{Q^{2}}}} \geq 4, \quad D=4 .
$$

Thus the fractal dimension of four dimensional quantum spacetime is also generally greater than its classical value.

The value of $Q^{2}$ is at present uncertain, principally because of the unknown infrared contribution of gravitons $Q_{\text {grav }}^{2}$ in (21), which is likely to be close to 8 [11]. If the total $Q^{2}<8$ we have argued in a previous letter [7] that the theory undergoes a BKT-like transition to a phase dominated by a dense 'gas' of long extruded structures, similar to the branched polymer phase seen in both the $D=2$ (for $c_{m}>1$ ) and $D=4$ simplicial simulations [12]. In that case the typical spacetimes become so irregular that apparently not even a fractal Hausdorff dimension adequately describes them. This is reflected in the square root in the denominator of (29) becoming imaginary for $Q^{2}<8$. At $Q^{2}=8, d_{H}=4(1+\sqrt{2})$ while

$$
d_{H} \rightarrow 4+\frac{16}{Q^{2}}+\mathcal{O}\left(Q^{-4}\right) \quad \text { as } \quad Q^{2} \rightarrow \infty,
$$

Thus the fractal dimension approaches its classical value in the limit in which the fluctuations of the conformal factor are suppressed. The behavior of the Hausdorff dimension as a function of $Q^{2}$ for $Q^{2} \geq 8$ is shown in Fig. 1 .

Screening of the Cosmological and Inverse Newtonian Couplings.

The quantum fluctuations of the conformal factor which give rise to the nontrivial Hausdorff dimension are also responsible for gravitational 'dressing' of the volume and Einstein terms in the effective action. The classical expressions for $S_{0}$ and $S_{2}$ are replaced by corresponding operators $\mathcal{O}_{\bar{\Delta}}$ with well-defined scaling dimensions $\beta_{0}$ and $\beta_{2}$ of (25) for $\bar{\Delta}=0$ and 2 respectively. The corresponding couplings $\lambda=\Lambda / 8 \pi G_{N}$ and $\kappa^{-1}=\left(8 \pi G_{N}\right)^{-1}$ must scale inversely with the volume in order for these terms in the effective action to be strictly marginal deformations at the conformal fixed point. This is a consequence of the consistency 
condition any covariant theory of gravity must satisfy when the quantum fluctuations of the conformal factor are considered.

Since $\Lambda / G_{N}$ multiplies the volume it must scale like

$$
\frac{\Lambda}{G_{N}} \sim V^{-1}
$$

Since $\left(8 \pi G_{N}\right)^{-1}$ multiplies an operator with conformal weight $\beta_{2}$ its finite volume scaling is

$$
G_{N} \sim V^{\frac{\beta_{2}}{\beta_{0}}} \equiv V^{\delta}
$$

We note that in this case the operator which becomes $S_{2}$ in the classical limit $Q^{2} \rightarrow \infty$ also acquires an additive renormalization at the fixed point, as discussed in refs. [4, 7].

The finite volume scaling relations (31) and (32) are predictions for $4 \mathrm{D}$ simplicial simulations, but are not yet directly relevant for continuum physics since the units in which the volume $V$ is measured have not been specified. Hence it is always possible to absorb this scaling of dimensionful quantities into a constant shift in $\sigma$. For a meaningful comparison a dimensionless ratio of two quantities having zero naive engineering dimensions should be formed so that one is measured in units of the other. Such a quantity is the dimensionless coupling,

$$
G_{N} \Lambda \sim V^{2 \delta-1} \sim \ell^{d_{H}(2 \delta-1)}
$$

upon incorporating our previous result for the Hausdorff dimension. This relation measures the cosmological term in units of the Planck mass. Since we have from (25) and (32),

$$
2 \delta-1=\frac{\sqrt{1-\frac{8}{Q^{2}}}-\sqrt{1-\frac{4}{Q^{2}}}}{1+\sqrt{1-\frac{4}{Q^{2}}}}<0
$$

for $Q^{2}>8$, the effective cosmological 'constant' in units of the Planck mass decreases at large distances, and $G_{N} \Lambda \rightarrow 0$ at the infrared fixed point in the infinite volume limit. Thus the quantum fluctuations of the conformal factor in $4 \mathrm{D}$ gravity provides a mechanism for the effective screening of the cosmological coupling at large distances, independently of its value from microscopic physics.

One may also compare the running of the Newtonian and cosmological couplings to a length scale fixed by a matter field operator, such as $\int d^{4} x \sqrt{g} \bar{\psi} \psi$. Since the fermion field has dimension $\frac{3}{2}$ this operator gets gravitationally dressed by the exponent $\beta_{3}$. Converting the finite volume scaling of this operator according to (24) to its corresponding coupling, we find that the fermion mass $m$ scales with the volume like

$$
m \sim V^{-\frac{\beta_{3}}{\beta_{0}}}
$$

This provides an independent standard of length against which we can measure the running of $G_{N}$ and $\Lambda$. Then we find

$$
\begin{aligned}
& G_{N} m^{2} \sim V^{\delta-2 \frac{\beta_{3}}{\beta_{0}}}=V^{\frac{\beta_{2}-2 \beta_{3}}{\beta_{0}}} \sim \ell^{d_{H} \frac{\beta_{2}-2 \beta_{3}}{\beta_{0}}} \\
& \frac{\Lambda}{G_{N} m^{4}} \sim V^{-1+4 \frac{\beta_{3}}{\beta_{0}}} \sim \ell^{d_{H} \frac{4 \beta_{3}-\beta_{0}}{\beta_{0}}} .
\end{aligned}
$$


Since

$$
d_{H} \frac{\beta_{2}-2 \beta_{3}}{\beta_{0}}=2\left(1+\sqrt{1+\frac{8}{Q^{2}}}\right)\left[\frac{1}{1+\sqrt{1-\frac{4}{Q^{2}}}}-\frac{1}{1+\sqrt{1-\frac{2}{Q^{2}}}}\right]>0
$$

for $Q^{2}>8$ the Newtonian 'constant' increases at large distances. This implies that the gravitational attractive force between two fermions falls more slowly than the classical Newtonian $r^{-2}$ force.

On the other hand since

$$
d_{H} \frac{4 \beta_{3}-\beta_{0}}{\beta_{0}}=4\left(1+\sqrt{1+\frac{8}{Q^{2}}}\right)\left[\frac{1}{1+\sqrt{1-\frac{2}{Q^{2}}}}-\frac{1}{1+\sqrt{1-\frac{8}{Q^{2}}}}\right]<0
$$

for $Q^{2}>8$ we conclude that the the cosmological 'constant' measured in units of the fermion mass decreases and is effectively screened at large distances.

For large $Q^{2}$ the large distance power laws in all these cases simplify and we have

$$
\begin{aligned}
G_{N} \Lambda & \sim \ell^{-\frac{4}{Q^{2}}} \\
G_{N} m^{2} & \sim \ell^{+\frac{1}{Q^{2}}} \\
\frac{\Lambda}{G_{N} m^{4}} & \sim \ell^{-\frac{6}{Q^{2}},} \quad Q^{2} \gg 8
\end{aligned}
$$

We emphasize that all these scaling relations, and in particular their dependence on the power $Q^{2}$ given by (21) have been derived using the anomalous dimensions that apply at the infrared fixed point of the conformal factor. Because the fluctuations of $\sigma$ which give rise to these anomalous dimensions are suppressed inside the scale of the horizon, the screening effects described here should become evident only at the very largest scales of the universe, comparable to the horizon scale, of order 1000 Mpc or greater.

\section{Consequences for Cosmology.}

The effective screening of the cosmological 'constant' at large distances suggests that it may be possible to construct a cosmological model in which the vacuum energy component runs continuously to smaller values as the universe expands. Consider a FRW model with expansion determined by a scale factor with an arbitrary power, $\nu$. Then the physical geodesic length $\ell$ of the previous treatment should scale like

$$
\ell \sim a(t) \sim t^{\nu}
$$

From (33) this implies that the vacuum energy density $\rho_{\Lambda}$ scales like

$$
\rho_{\Lambda} \equiv \frac{\Lambda}{8 \pi G_{N}} \sim M_{p l}^{4} t^{\nu d_{H}(2 \delta-1)}
$$

when measured in Planck units (hereafter fixed). For any $\nu$ the Hubble 'constant' scales as

$$
H(t) \equiv \frac{\dot{a}}{a} \sim t^{-1}
$$


so if Einstein's equations hold, or less stringently, if the Ricci scalar remains proportional to the cosmological term in the effective equations of motion, then we should have

$$
R(t) \sim H^{2}(t) \sim t^{-2} \sim G_{N} \rho_{\Lambda} \sim M_{p l}^{2} t^{\nu d_{H}(2 \delta-1)} .
$$

Therefore the expansion power of the scale factor in the cosmological model is determined by the previous considerations to be

$$
\nu=\frac{2}{d_{H}(1-2 \delta)}=\frac{1}{2} \frac{1-\sqrt{1+\frac{8}{Q^{2}}}}{1+\sqrt{1-\frac{8}{Q^{2}}}-2 \sqrt{1-\frac{4}{Q^{2}}}} \geq \frac{1}{2} .
$$

This power equals $\frac{1}{2}$ at $Q^{2}=8$, and goes to infinity linearly as $Q^{2} \rightarrow \infty$. The latter is the classical limit in which the fluctuations of the conformal factor are suppressed and we recover de Sitter spacetime with its exponential expansion. The behavior of $\nu$ as a function of $Q^{2}$ is illustrated in Fig. 2.

This power law model has some interesting consequences. First, it implies that for any $Q^{2}$ the vacuum energy density will always be a finite fraction of the critical density,

$$
\Omega_{\Lambda} \sim \frac{\rho_{\Lambda}(t)}{H^{2}(t)}=\text { constant }
$$

which one would generically expect to be of order unity. Indeed, if we write

$$
\Lambda(t)=k M_{p l}^{2}\left(\frac{t_{p l}}{t}\right)^{2}
$$

and estimate the present age of the universe at $t_{0} \simeq 12 \mathrm{Gyr}$, and the present Hubble parameter $H_{0}=75 \mathrm{~km} / \mathrm{sec} / \mathrm{Mpc}$ we obtain

$$
\Lambda_{0} \simeq 2.05 k \times 10^{-122} M_{p l}^{2}
$$

and

$$
\Omega_{\Lambda}=\Lambda_{0} / 3 H_{0}^{2} \simeq 0.40 k
$$

which for $k$ of order unity is of order of the present bounds on the cosmological term. If $k=1.5$ then $\Omega_{\Lambda}=0.6$ as suggested by a number of recent papers in the astrophysics literature [13]. This would make it possible to have an asymptotically spatially flat cosmological model with $\Omega_{\text {total }}=1$. We also remark that if $\nu>\frac{2}{3}$ this would imply an older age for the universe than that inferred from classical matter dominated cosmology.

The second interesting aspect of the power (44) is that it rises very rapidly from its minimum value of $\frac{1}{2}$, reaching $\frac{2}{3}$ at $Q^{2} \simeq 8.111$ and is greater than unity if $Q^{2}>Q_{1}^{2} \simeq 8.636$. Since $Q^{2}$ depends on the number of massless fields through (21) and 'massless' in this context means light compared to $H(t)$, the effective value of $Q^{2}$ could very well have been much higher in the early universe than it is in the present epoch. A value of $Q^{2}>Q_{1}^{2}$ implies power law inflation, which if it persists for a long enough time removes the 'horizon problem' of the classical FRW cosmology. Since the universe becomes arbitrarily flat at late times in a 
model with scaling behavior given by (43) and (45) there is no 'flatness problem' in such a model. Of course, a full cosmological model incorporating the scaling behavior conjectured here (and free of any new problems) must be constructed to realize this dynamical solution of the cosmological 'constant' problem.

As the universe expands and ages each light but not strictly massless degree of freedom will have its Compton wavelength become of order of the horizon at a different time, when we must expect the possibility that the 'constant' in (45) might change, analogous to the changes in a renormalization group beta function as kinematic mass thresholds are crossed. The detailed physics of these transition eras would fix the value of $\Omega_{\Lambda}$ during the next epoch of power law expansion. Finally, from perturbative estimates and numerical simulations there is reason to believe that the present value of $Q^{2}$ could be close to (but presumably slightly larger than) 8. If that is the case then the present value of the power $\nu$ is somewhat larger than $\frac{1}{2}$, and perhaps close to the $\frac{2}{3}$ predicted by standard matter dominated cosmology (cf. Fig. 2).

Globally, of course, a cosmological model constructed along the lines of the foregoing speculations would be quite different, and observationally distinguishable from the standard model. First, the presence of the effective cosmological term would be expected to modify the linear Hubble relation at the very largest scales, which is a subject of very active current interest. Secondly, because of the increase of the Newtonian coupling at the largest scales according to (36), estimates of mass through virial arguments could be somewhat larger than the actual mass present in large clusters and superclusters of galaxies. Taking $Q^{2} \simeq 8$ the power in (36) and (37) is approximately 0.24 . Since $10^{0.24} \simeq 1.74$ the increase of the Newtonian coupling over one decade of distance would lead to a $74 \%$ increase in the dark 'matter' attributed to the supercluster by virial estimates, on top of the effect of the homogenous vacuum density of $\rho_{\Lambda}$. Together, these two effects may eliminate the need to invoke exotic forms of dark matter to close the universe, so this is a kind of null prediction. In addition to these effects, since the spectral index of the initial adiabatic density fluctuations is also altered by the infrared fixed point of the conformal factor [8], models of structure formation would have to be reconsidered $a b$ initio. Finally, the most radical departure from the standard model arises from the fractal structure of spacetime itself at the largest scales, which if correct, implies that the universe and the matter distributed in it are not homogeneous, even at scales approaching the horizon scale.

\section{Acknowledgments}

The authors wish to acknowledge NATO grant Collaborative Research Grant 971650 for making this work possible. P. O. M. and E. M. also wish to thank the Centre de Physique Théorique at the Ecole Polytechnique for its hospitality. 


\section{REFERENCES}

[1] V. G. Knizhnik, A. M. Polyakov, and A. B. Zamolodchikov, Mod. Phys. Lett. A3 (1988) 819 ;

F. David, Mod. Phys. Lett. A3 (1988) 1651;

J. Distler and H. Kawai, Nucl. Phys. B321 (1989) 509.

[2] F. David, Nucl. Phys. B257 (1985) 45;

V. A. Kazakov, Phys. Lett. B150 (1985) 282;

J. Ambjørn, B. Durhuus, J. Frohlich and P. Orland, Nucl. Phys. B270 (1986) 457;

A. Billoire and F. David, Nucl. Phys. B275 (1986) 617;

D. V. Boulatov, V. A. Kazakov, I. K. Kostov and A. A. Migdal, Nucl. Phys. B275 (1986) 641 ;

J. Ambjørn and J. Jurkiewicz, Phys. Lett. B278 (1992) 42;

M.E. Agishtein and A.A. Migdal, Mod. Phys. Lett. A7 (1992) 1039;

S. M. Catterall, J. B. Kogut, and R. L. Renken, Phys. Rev. D45 (1992) 2957.

[3] R. J. Riegert, Phys. Lett. B134 (1984) 56;

E. S. Fradkin and A. A. Tseytlin, Phys. Lett. B134 (1984) 187;

I. L. Buchbinder, S. D. Odintsov and I. L. Shapiro, Phys. Lett. B162 (1985) 93;

E. T. Tomboulis, Nucl. Phys. B329 (1990) 410;

S. D. Odintsov and I. L. Shapiro, Class. Quant. Grav. 8 (1991) L57;

S. D. Odintsov, Z. Phys. C54 (1992) 531.

[4] I. Antoniadis and E. Mottola, Phys. Rev. D45 (1992) 2013.

[5] J. W. York, Phys. Rev. Lett. 26 (1971) 1656; ibid. 28 (1972) 1082; Jour. Math. Phys. 13 (1972) 125; ibid. 14 (1973) 125.

[6] I. Antoniadis, P. O. Mazur and E. Mottola, Phys. Rev. D55 (1997) 4756; ibid. 4770.

[7] I. Antoniadis, P. O. Mazur and E. Mottola, Phys. Lett. B323 (1994) 284; B394 (1997) 49.

[8] I. Antoniadis, P. O. Mazur and E. Mottola, Phys. Rev. Lett. 79 (1997) 14.

[9] N. Kawamoto, Phys. Rev. Lett. 68 (1992) 2113;

N. Kawamoto, V. A. Kazakov, Y. Saeki, and Y. Watabiki, Nucl. Phys. B26(Proc. Suppl.) (1992) 584;

Y. Watabiki, Prog. Theor. Phys. Suppl. 114 (1993) 1; e-print archive, hep-th/9605185.

[10] S. Catterall, G. Thorleifsson, M. Bowick, and V. John, Phys. Lett. B354 (1995) 58;

J. Ambjørn and K. N. Anagnostopoulos, Nucl. Phys. B497 (1997) 445;

J. Ambjørn, K. N. Anagnostopoulos, and G. Thorliefsson, e-print archive, hep-lat/9709025.

[11] I. Antoniadis, P. O. Mazur and E. Mottola, Nucl. Phys. B388 (1992) 627.

[12] J. Ambjørn, S. Jain, J. Jurkiewicz and C. F. Kristjansen, Phys. Lett. B305 (1993) 208;

S. M. Catterall, J. B. Kogut, and R. L. Renken, Phys. Lett. B328 (1994) 277;

B. De Bakker and J. Smit, Nucl. Phys. B439 (1995) 239;

J. Ambjørn and J. Jurkiewicz, Nucl. Phys. B451 (1995) 643;

J. Ambjørn, J. Jurkiewicz, and Y. Watabiki, Jour. Math. Phys. 36 No. 5 (1995) 6299.

[13] Y. Mellier et. al., e-print archive, astro-ph/9609197;

A. G. Riess et. al., e-print archive, astro-ph/9805201. 


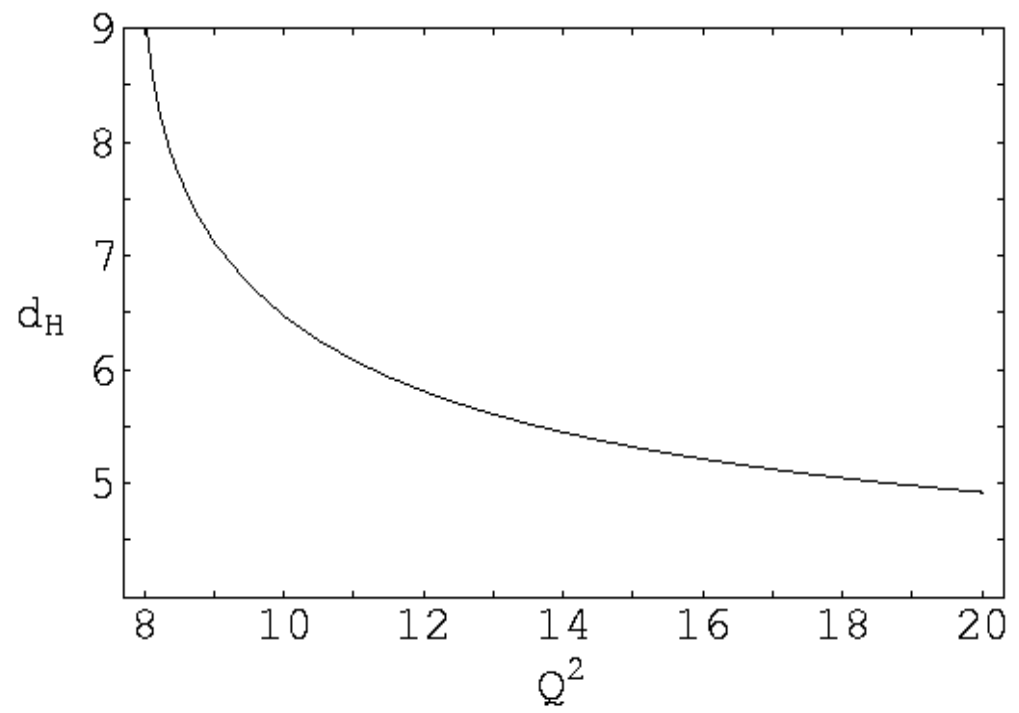

FIG. 1. The fractal Hausdorff dimension $d_{H}$, Eqns. (1) and (29), as a function of the anomaly coefficient $Q^{2}$.

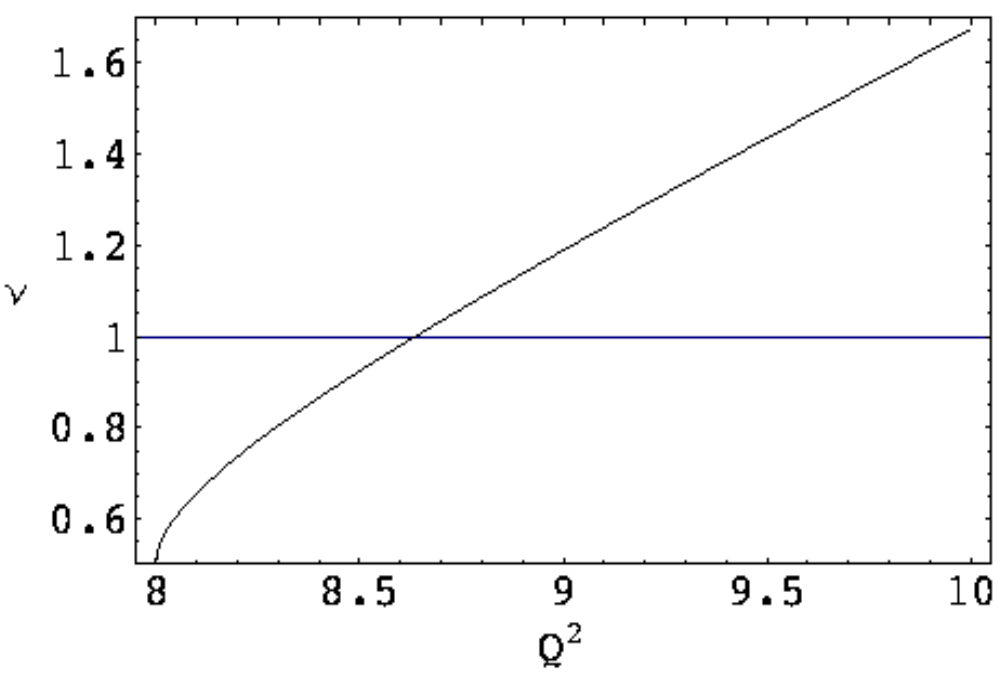

FIG. 2. The power law coefficient of the expansion rate of the universe, Eqns. (40) and (44) as a function of the anomaly coefficient $Q^{2}$. 